K. Yamamuro

Nagoya Math. J.

Vol. 149 (1998), 19-32

\title{
ON TRANSIENT MARKOV PROCESSES OF ORNSTEIN-UHLENBECK TYPE
}

\author{
KOUJI YAMAMURO
}

\begin{abstract}
For Hunt processes on $\mathbf{R}^{d}$, strong and weak transience is defined by finiteness and infiniteness, respectively, of the expected last exit times from closed balls. Under some condition, which is satisfied by Lévy processes and Ornstein-Uhlenbeck type processes, this definition is expressed in terms of the transition probabilities. A criterion is given for strong and weak transience of Ornstein-Uhlenbeck type processes on $\mathbf{R}^{d}$, using their Lévy measures and coefficient matrices of linear drift terms. An example is discussed.
\end{abstract}

\section{§1. Introduction}

Let $\mathbf{R}^{d}$ be the $d$-dimensional Euclidean space and $\mathcal{B}$ be the Borel $\sigma$ algebra in $\mathbf{R}^{d}$. We denote the inner product and the norm in $\mathbf{R}^{d}$ by $\langle x, y\rangle=\sum_{j=1}^{d} x_{j} y_{j}$ and $|x|=\sqrt{\langle x, x\rangle}$, respectively, for $x=\left(x_{j}\right)_{1 \leq j \leq d}$, $y=\left(y_{j}\right)_{1 \leq j \leq d}$. Let $X=\left(\Omega, \mathcal{M}, \mathcal{M}_{t}, X_{t}, \theta_{t}, P^{x}\right)$ be a Markov process (in the sense of [1]) with state space $\left(\mathbf{R}^{d}, \mathcal{B}\right)$, satisfying $P^{x}\left(X_{0}=x\right)=1$. Assume that $X$ is a Feller process. Here we say that $X$ is a Feller process if it satisfies the following properties: for any continuous function $f$ on $\mathbf{R}^{d}$ vanishing at infinity,

(i) $P_{t} f$ is continuous for all $t>0$,

(ii) $P_{t} f \rightarrow f$ uniformly as $t \rightarrow 0$,

where $P_{t}$ is the transition operator of $X$. This process $X$ is a Hunt process by virtue of Theorem 9.4 in [1] p.46. The process $X$ is called transient if it satisfies

$$
P^{x}\left(\lim _{t \rightarrow \infty}\left|X_{t}\right|=\infty\right)=1
$$

for every $x \in \mathbf{R}^{d}$. Let $L_{B}=\sup \left\{t \geq 0: X_{t} \in B\right\}$ for any $B \in \mathcal{B}$, which is called the last exit time from $B$, where the supremum of the empty set is 0 . Let $T_{B}=\inf \left\{t>0: X_{t} \in B\right\}$ for any $B \in \mathcal{B}$. Now we define strong and weak transience.

Received May 21, 1996. 
Definition 1. Let $X$ be transient. Then $X$ is called strongly transient if it satisfies

$$
E^{x} L_{B}<\infty
$$

for every closed ball $B$ and every $x$. In the remaining case it is called weakly transient.

Our definition of strong and weak transience is different in appearance from that of Port [3] given for irreducible Markov chains. But in [3] he showed that strong and weak transience is equivalent to finiteness and infiniteness, respectively, of the expectation of the last exit times from finite sets. Hence the definition above is a natural extension.

Now we introduce the following condition:

$$
\inf _{x \in K} E^{x} e^{-L_{K}}>0 \quad \text { for any compact set } K .
$$

Our first result is the following theorem.

THEOREM 1.1. Let $X$ be transient and satisfy the condition (1.1). Then $X$ is strongly transient if and only if we have, for any $x$ and any closed ball $K$,

$$
\int_{0}^{\infty} d t t P^{x}\left(X_{t} \in K\right)<\infty .
$$

In particular we shall investigate transient Ornstein-Uhlenbeck type processes. An Ornstein-Uhlenbeck type process ( OU type process ) $X$ is a Feller process with state space $\left(\mathbf{R}^{d}, \mathcal{B}\right)$ such that the process $\left\{X_{t}\right\}$ under the probability measure $P^{x}$ has the same finite-dimensional distribution as the process $\left\{\bar{X}_{t}\right\}$ defined by

$$
\bar{X}_{t}=e^{-t Q} x+\int_{0}^{t} e^{-(t-u) Q} d Z_{u}
$$

where $\left\{Z_{t}\right\}$ is a Lévy process, and $Q$ is a real $d \times d$-matrix of which all eigenvalues have positive real parts. Here the stochastic integral with respect to the Lévy process is defined by convergence in probability from integrals of simple functions. This process $X$ is called the OU type process associated with $\left\{Z_{t}\right\}$ and $Q$. The infinitesimal generator of $X$ is given in [6] and [7]. Let the Lévy process $\left\{Z_{t}\right\}$ have the following characteristic function:

$$
E \exp \left(i\left\langle z, Z_{t}\right\rangle\right)=\exp (t \psi(z))
$$




$$
\psi(z)=-2^{-1}\langle z, B z\rangle+\int_{\mathbf{R}^{d}}\left(e^{i\langle z, x\rangle}-1-i\langle z, x\rangle 1_{\{|x|<1\}}(x)\right) \rho(d x)+i\langle b, z\rangle,
$$

where $B$ is a symmetric nonnegative-definite constant matrix, $\rho$ is a measure on $\mathbf{R}^{d}$ with $\rho(\{0\})=0$ and $\int\left(1 \wedge|y|^{2}\right) \rho(d y)<\infty, b$ is a constant vector, and $1_{\{|x|<1\}}$ is the indicator function of the set $\{|x|<1\}$. The measure $\rho$ is called Lévy measure.

Recurrence criterion of OU type processes was found by T. Shiga [9] in one dimension, by K. Sato, T. Watanabe, and M. Yamazato [7] and by K. Sato, T. Watanabe, K. Yamamuro, and M. Yamazato [8] in some multidimensional cases, and finally by $\mathrm{T}$. Watanabe [10] in the general multidimensional case.

Our second result is the following criterion of strong and weak transience for OU type processes.

TheOREM 1.2. Let $X$ be a transient OU type process. Fix $c>0$. Then $X$ is strongly transient if and only if

$$
\int_{0}^{\infty} t \exp \left[\int_{0}^{t} d s \int_{|x| \geq c}\left(e^{-\left|e^{-s Q} x\right|}-1\right) \rho(d x)\right] d t<\infty
$$

\section{$\S 2$. Proof of Theorem 1}

In order to prove Theorem 1 , we need the following lemmas. For a nonnegative measurable function $f$ on $\mathbf{R}^{d}$, define $U^{\lambda} f$ by

$$
U^{\lambda} f(x)=\int_{0}^{\infty} e^{-\lambda t} P_{t} f(x) d t
$$

Let us denote $U=U^{0}$.

LEMma 2.1. Let $X$ be transient and satisfy the condition (1.1). Then $U 1_{K}$ is bounded for any compact set $K$.

Proof. In [2] this lemma was proved under some other condition. Let $K_{n}$ be a sequence of compact sets such that $K_{n}$ is contained in the interior of $K_{n+1}$ and $\cup_{n} K_{n}=\mathbf{R}^{d}$. Let us denote $g_{n}(x)=P^{x}\left(T_{K_{n}}<\infty\right)$. We denote $g_{n, k}=k\left(g_{n}-P_{\frac{1}{k}} g_{n}\right)$. We notice that $U g_{n, k}<\infty$. By virtue of Lemma 3.1 in [2] p.403, we have, for any $m>1$,

$$
g_{n}=\lim _{k \rightarrow \infty} \uparrow U g_{n, m^{k}} .
$$


Here the right-hand side denotes the increasing limit of $U g_{n, m^{k}}$ as $k \rightarrow \infty$. Furthermore,

$$
1=\lim _{n \rightarrow \infty} \uparrow g_{n}=\lim _{n \rightarrow \infty} \uparrow U g_{n, m^{n}}
$$

Define $g^{m}$ by

$$
g^{m}=\sum_{n=1}^{\infty} \frac{g_{n, m^{n}}}{m^{n} 2^{n}} .
$$

From $g_{n, m^{n}} \leq m^{n}$ we have $g^{m} \leq 1$. And from (2.1.2) we have $0<U g^{m} \leq 1$. Set $h=U^{1} g^{m}$. Then $h \leq U g^{m} \leq 1$. From the resolvent equation we have $U h \leq U g^{m} \leq 1$. Since $U g^{m}>0$, we get $h>0$. Furthermore, since $T_{K_{n}} \circ \theta_{t}<\infty$ and $L_{K_{n}}>t$ are equivalent,

$$
\begin{aligned}
& U^{1} g^{m}(x) \\
= & \sum_{n=1}^{\infty} \frac{1}{2^{n}} \int_{0}^{\infty} e^{-t}\left(P^{x}\left(T_{K_{n}} \circ \theta_{t}<\infty\right)-P^{x}\left(T_{K_{n}} \circ \theta_{\frac{1}{m^{n}}+t}<\infty\right)\right) d t \\
= & \sum_{n=1}^{\infty} \frac{1}{2^{n}}\left[E^{x}\left(1-e^{-L_{K_{n}}}\right)-e^{\frac{1}{m^{n}}} E^{x}\left(e^{-\frac{1}{m^{n}}}-e^{-L_{K_{n}}}: L_{K_{n}}>\frac{1}{m^{n}}\right)\right] \\
\geq & \sum_{n=1}^{\infty} \frac{1}{2^{n}}\left(e^{\frac{1}{m^{n}}}-1\right) E^{x}\left[e^{-L_{K_{n}}}: L_{K_{n}}>\frac{1}{m^{n}}\right] \\
= & \sum_{n=1}^{\infty} \frac{1}{2^{n}}\left(e^{\frac{1}{m^{n}}}-1\right) e^{-\frac{1}{m^{n}}} E^{x}\left[E^{X} \frac{1}{m^{n}}\left[e^{-L_{K_{n}}}: L_{K_{n}}>0\right]\right] .
\end{aligned}
$$

Now there is a compact set $K_{n-2}$ such that $K \subset K_{n-2}$. We can choose a nonnegative continuous function $f$ such that the support of $f$ is $K_{n-1}$, $f \leq 1$, and $f=1$ on $K_{n-2}$. Then, for sufficiently large $m$,

$$
\begin{aligned}
& E^{x}\left[E^{X} \frac{1}{m^{n}}\left[e^{-L_{K_{n}}}: L_{K_{n}}>0\right]\right] \\
& \geq E^{x}\left[f\left(X_{\frac{1}{m^{n}}}\right)\right] \inf _{y \in K_{n-1}} E^{y}\left[e^{-L_{K_{n}}}: L_{K_{n}}>0\right] \\
& \geq\left(f(x)-\frac{1}{2}\right) \inf _{y \in K_{n-1}} E^{y} e^{-L_{K_{n}}} .
\end{aligned}
$$

Here we got the last inequality by using the Feller property. Hence, using the condition (1.1), we have $\inf _{x \in K} h(x)>0$ from $f=1$ on $K_{n-2}$. From this fact we get

$$
U 1_{K} \leq \frac{U h}{\inf _{x \in K} h(x)} \leq \frac{1}{\inf _{x \in K} h(x)}
$$


concluding the proof.

LEMMA 2.2. Let $X$ be transient and satisfy the condition (1.1). If $B$ and $C$ are compact sets such that the interior of $B$ contains $C$, then there are positive constants $\alpha$ and $\beta$ such that

$$
\alpha P^{x}\left(T_{C}<\infty\right) \leq U 1_{B}(x) \leq \beta P^{x}\left(T_{B}<\infty\right)
$$

for every $x$.

Proof. Using the strong Markov property, we have

$$
\begin{aligned}
U 1_{B}(x) & \geq E^{x}\left[E^{X_{T_{C}}}\left[\int_{0}^{\infty} 1_{B}\left(X_{t}\right) d t\right]: T_{C}<\infty\right] \\
& \geq P^{x}\left(T_{C}<\infty\right) \inf _{x \in C} U^{\lambda} 1_{B}(x),
\end{aligned}
$$

where $0<\lambda<\infty$. Since $X$ is a Feller process, we have $\inf _{x \in C} U^{\lambda} 1_{B}(x)>0$. Hence we obtain the first inequality in (2.2.1). Next we shall prove the second inequality. We have

$$
\begin{aligned}
U 1_{B}(x) & =E^{x}\left[E^{X_{T_{B}}}\left[\int_{0}^{\infty} 1_{B}\left(X_{t}\right) d t\right]: T_{B}<\infty\right] \\
& \leq P^{x}\left(T_{B}<\infty\right) \sup _{x \in B} U 1_{B}(x) .
\end{aligned}
$$

Using Lemma 2.1, we have $\sup _{x \in B} U 1_{B}(x)<\infty$. This shows the Lemma.

Proof of Theorem 1. We have

$$
E^{x} L_{B}=\int_{0}^{\infty} P^{x}\left(T_{B} \circ \theta_{t}<\infty\right) d t .
$$

Let $A$ be a closed ball such that $B$ is contained in the interior of $A$. By virtue of Lemma 2.2, we get

$$
\begin{aligned}
\frac{1}{\alpha} \int_{0}^{\infty} t P_{t}(x, A) d t & =\frac{1}{\alpha} \int_{0}^{\infty} P_{t} U 1_{A}(x) d t \\
& \geq E^{x} L_{B} \\
& \geq \frac{1}{\beta} \int_{0}^{\infty} P_{t} U 1_{B}(x) d t=\frac{1}{\beta} \int_{0}^{\infty} t P_{t}(x, B) d t .
\end{aligned}
$$

Hence,

$$
E^{x} L_{B}<\infty \quad \text { for any closed ball } B
$$


if and only if

$$
\int_{0}^{\infty} t P_{t}(x, B) d t<\infty \quad \text { for any closed ball } B .
$$

We have thus proved the theorem.

Remark 2.3. If $X$ is a transient OU type process or a transient Lévy process, it satisfies the condition (1.1). Because, for any compact set $K$, there is a compact set $C$ such that $E^{x} e^{-L_{K}} \geq E^{0} e^{-L_{C}}$ for all $x \in K$. Further (1.1) holds in case of transient strong Feller processes. Here we say that $X$ is a strong Feller process if it is a Feller process and if (i) in the Feller property holds for any bounded measurable function $f$. In fact, we have $E^{x} \exp \left\{-L_{K}\right\} \geq E^{x} \exp \left\{-\left(L_{K} \circ \theta_{t}+t\right)\right\}=e^{-t} P_{t} E^{x} \exp \left\{-L_{K}\right\}$, and the last expression is continuous in $x$ by virtue of the strong Feller property. Since $E^{x} \exp \left\{-L_{K}\right\}>0$ for all $x \in K$, we have the condition (1.1).

Remark 2.4. Let

$$
r(t)=\int_{t}^{\infty} d s \int d x h(x) \int P^{x}\left(X_{s} \in d y\right) h(y),
$$

where $h$ is a nonnegative continuous function on $\mathbf{R}^{d}$ with compact support, not identically zero. In [4] the definition of strong and weak transience for Lévy processes is given by $\int_{0}^{\infty} r(t) d t<\infty$ and $=\infty$, respectively. In case of an OU type process, since the condition of Theorem 1 is satisfied by virtue of Remark 2.3, we see that it is strongly transient if and only if $\int_{0}^{\infty} r(t) d t<\infty$.

\section{§. Proof of Theorm 2}

In this section let $X$ be an OU type process. First we shall state a fundamental lemma discovered by $T$. Watanabe [10] on boundedness of some integrals. This will play an important role in the proof of Theorem 2 . Let $n$ and $m$ be positive integers. The number $\bar{z}$ signifies the complex conjugate of $z$. Let $\gamma_{j}(1 \leq j \leq n)$ be complex numbers. Let $P_{j}(s), 1 \leq j \leq$ $n$, be polynomials with degrees being at most $m$. We assume that $P_{j}(s)$ has real coefficients if $\gamma_{j}$ is real, and that $\gamma_{j}=\overline{\gamma_{k}}$ and $P_{j}(s)=\overline{P_{k}(s)}$ for some $k$ if $\gamma_{j}$ is not real. Define a function $f(s)$ on $\mathbf{R}^{1}$ as

$$
f(s)=\sum_{j=1}^{n} e^{\gamma_{\jmath} s} P_{j}(s)
$$


Further let $I(x)$ be a real bounded measurable function on $\mathbf{R}^{1}$ and let $J(x)=\int_{0}^{x} I(u) d u$.

Lemma 3.1. (T. Watanabe [10]) Suppose that

$$
\sup _{x \in \mathbf{R}^{1}}|I(x)| \leq 1 \quad \text { and } \quad \sup _{x \in \mathbf{R}^{1}}|J(x)| \leq 1
$$

(i) We obtain

$$
\left|\int_{0}^{N} I(f(s)) d s\right| \leq K_{1}+K_{2} \log \left(\frac{1}{|f(0)|} \vee 1\right) \quad \text { for every } N>0
$$

(ii) In addition, suppose that

$$
\sup _{x \neq 0} \frac{|I(x)|}{|x|} \leq 1
$$

Then we obtain

$$
\left|\int_{M}^{N} I(f(s)) d s\right| \leq K_{3} \text { for every } M \text { and } N \text { with } M<N .
$$

Here $K_{1}, K_{2}$, and $K_{3}$ are positive constants depending only on $m, n$, and $\left\{\gamma_{j}\right\}_{j=1}^{n}$.

For $c>0$ we denote the restriction of $\rho$ to the set $\{x:|x| \geq c\}$ by $\rho_{c}$. Let $\left\{Z_{t}^{c}\right\}$ be a compound Poisson process with Lévy measure $\rho_{c}$. The process $X^{c}$ denotes the OU type process associated with $\left\{Z_{t}^{c}\right\}$ and $Q$.

LEMmA 3.2. Let $X$ be transient. If $X^{c}$ is weakly transient for some $c>0$, then $X$ is weakly transient.

Proof. Choose a Lévy process $\left\{W_{t}\right\}$ independent of $\left\{Z_{t}^{c}\right\}$ such that $\left\{W_{t}+Z_{t}^{c}\right\}$ and $\left\{Z_{t}\right\}$ have common finite-dimensional distributions. Let $Y$ be the OU type process associated with $\left\{W_{t}\right\}$ and $Q$. Then, identifying $X^{c}$ and $Y$ with $\bar{X}_{t}$ and $\bar{Y}_{t}$ defined similarly to $\bar{X}_{t}$ in section 1 , we see that the processes $X^{c}$ and $Y$ are independent. We notice that $Y$ has a limit distribution $\mu$, because the Lévy measure $\rho-\rho_{c}$ satisfies $\int_{|x| \geq 1} \log |x|(\rho-$ $\left.\rho_{c}\right)(d x)<\infty$ (see [6]). By Theorem 1 and Remark 2.3, we can choose closed balls $E_{1}$ and $E_{2}$ such that $\int_{0}^{\infty} t P^{x}\left(X_{t}^{c} \in E_{1}\right) d t=\infty$ for some $x$ and $E_{2}$ is a 
$\mu$-continuity set with $\mu\left(E_{2}\right)>0$. Now let a closed ball $E$ contain $E_{1}+E_{2}$. Let $t$ be large enough. Then

$$
\begin{aligned}
P^{x}\left(X_{t} \in E\right) & =P^{0}\left(X_{t} \in E-e^{-t Q} x\right) \\
& \geq P^{0}\left(X_{t}^{c} \in E_{1}-e^{-t Q} x, Y_{t} \in E_{2}\right) \\
& \geq P^{0}\left(X_{t}^{c} \in E_{1}-e^{-t Q} x\right) \frac{\mu\left(E_{2}\right)}{2} \\
& \geq P^{x}\left(X_{t}^{c} \in E_{1}\right) \frac{\mu\left(E_{2}\right)}{2} .
\end{aligned}
$$

Hence we obtain $\int_{0}^{\infty} t P^{x}\left(X_{t} \in E\right) d t=\infty$, and this completes the proof.

Using these lemmas, we shall prove Theorem 2. Let $P_{t}(x, E)$ and $P_{t}^{c}(x, E)$ be transition probabilities of $X$ and $X^{c}$, respectively. And let $P_{t}$ and $P_{t}^{c}$ be their transition operators.

Proof of Theorem 2. For any $a>0$, the Fourier transform of $h_{a}(x)=$ $\Pi_{j=1}^{d}\left(\left(a-\left|x_{j}\right|\right) \vee 0\right)$ is expressed as

$$
\hat{h}_{a}(z)=\int e^{i\langle x, z\rangle} h_{a}(x) d x=\prod_{j=1}^{d} 4 z_{j}^{-2} \sin ^{2}\left(2^{-1} a z_{j}\right) .
$$

We have the inversion formula

$$
h_{a}(x)=\frac{1}{(2 \pi)^{d}} \int e^{-i\langle x, z\rangle} \hat{h}_{a}(z) d z .
$$

At first we suppose that $X$ is strongly transient. By virtue of Lemma 3.2, we obtain that $X^{c}$ is strongly transient for any $c$. Let $\hat{P}_{t}(x, z)$ and $\hat{P}_{t}^{c}(x, z)$ be the characteristic functions of $P_{t}(x, d y)$ and $P_{t}^{c}(x, d y)$, respectively. There is a positive constant $N$ satisfying that $\left|e^{-s Q} z\right| \leq N|z|$ for all $s>0$ and $z \in \mathbf{R}^{d}$. Choose $a$ large enough. We have $h_{a}\left(e^{-s Q} x+z\right) \geq h_{a-N|x|}(z)$. Then, from Theorem 1,

$$
\begin{aligned}
\infty & >\int_{0}^{\infty} d t t \int P_{t}^{c}(x, d y) h_{a}(y) \\
& =\int_{0}^{\infty} d t t \int P_{t}^{c}(0, d y) h_{a}\left(e^{-t Q} x+y\right) \\
& \geq \int_{0}^{\infty} d t t \int P_{t}^{c}(0, d y) h_{a-N|x|}(y)
\end{aligned}
$$




$$
\begin{aligned}
& =\frac{1}{(2 \pi)^{d}} \int_{0}^{\infty} d t t \int d z \hat{h}_{a-N|x|}(z) \hat{P}_{t}^{c}(0,-z) \\
& =\frac{1}{(2 \pi)^{d}} \int_{0}^{\infty} d t t \int d z \hat{h}_{a-N|x|}(z) \operatorname{Re} \hat{P}_{t}^{c}(0,-z) .
\end{aligned}
$$

We have

$$
\hat{P}_{t}^{c}(0, z)=\exp \left[\int_{0}^{t} d s \int\left(\exp \left(i\left\langle z, e^{-s Q} x\right\rangle\right)-1\right) \rho_{c}(d x)\right] .
$$

Hence we get

$$
\operatorname{Re} \hat{P}_{t}^{c}(0, z)=\cos F_{c}(t, z) \exp G_{c}(t, z)
$$

where

$$
\begin{gathered}
F_{c}(t, z)=\int_{0}^{t} d s \int \sin \left\langle z, e^{-s Q} x\right\rangle \rho_{c}(d x), \\
G_{c}(t, z)=\int_{0}^{t} d s \int\left(\cos \left\langle z, e^{-s Q} x\right\rangle-1\right) \rho_{c}(d x) .
\end{gathered}
$$

Use Lemma 3.1 (ii) for $I(x)=\sin x$. Then

$$
\left|F_{c}(t, z)\right| \leq K_{1} \rho(|x| \geq c)
$$

where $K_{1}$ is a constant depending only on $Q$. Choose $c$ so large that $K_{1} \rho(|x| \geq c)<\pi / 4$. Then $\cos F_{c}(t, z) \geq 1 / \sqrt{2}$. Therefore,

$$
\infty>\int_{0}^{\infty} d t t \int d z \hat{h}_{a-N|x|}(z) \exp G_{c}(t, z)
$$

We have $\hat{h}_{a-N|x|}(z)>0$ for $|z|<\frac{2 \pi}{a-N|x|}$. Hence, for some $z$ with $0<|z|<1$,

$$
\begin{aligned}
\infty> & \int_{0}^{\infty} d t \exp G_{c}(t, z) \\
& \int_{0}^{\infty} d t t \exp \left[\int_{0}^{t} d s \int\left(e^{-\left|\left\langle z, e^{-s Q} x\right\rangle\right|}-1\right) \rho_{c}(d x)+H_{c}(t, z)\right]
\end{aligned}
$$

where

$$
H_{c}(t, z)=\int_{0}^{t} d s \int\left(\cos \left\langle z, e^{-s Q} x\right\rangle-e^{-\left|\left\langle z, e^{-s Q} x\right\rangle\right|}\right) \rho_{c}(d x)
$$


Letting $I(x)=2^{-1}\left(\cos x-e^{-|x|}\right)$ in (ii) of Lemma 3.1, we have

$$
\begin{aligned}
\infty & >\int_{0}^{\infty} d t t \exp \left[\int_{0}^{t} d s \int\left(e^{-\left|\left\langle z, e^{-s Q} x\right\rangle\right|}-1\right) \rho_{c}(d x)\right] \\
& \geq \int_{0}^{\infty} d t t \exp \left[\int_{0}^{t} d s \int\left(e^{-\left|e^{-s Q} x\right|}-1\right) \rho_{c}(d x)\right] .
\end{aligned}
$$

Next we shall prove the converse. In order to prove that $X$ is strongly transient, it suffices to prove that, for all $x$ and for all small $a$,

$$
\int_{0}^{\infty} d t t \int P_{t}(x, d z) \hat{h}_{a}(z)<\infty .
$$

We have

$$
\begin{aligned}
\int P_{t}(x, d z) \hat{h}_{a}(z) & =\int d z h_{a}(z) \hat{P}_{t}(0, z) e^{i\left\langle e^{-t Q} x, z\right\rangle} \\
& \leq \int d z h_{a}(z)\left|\hat{P}_{t}(0, z)\right| \\
& \leq a^{d} \int_{|z|<a \sqrt{d}} d z\left|\hat{P}_{t}^{c}(0, z)\right|
\end{aligned}
$$

Hence it suffices to prove, for some $c>0$,

$$
\int_{0}^{\infty} d t t \int_{|z|<1} d z\left|\hat{P}_{t}^{c}(0, z)\right|<\infty .
$$

Furthermore,

$$
\begin{aligned}
& \int_{|z|<1}\left|\hat{P}_{t}^{c}(0, z)\right| d z \\
= & \int_{|z|<1} \exp G_{c}(t, z) d z \\
= & \exp \left[\int_{0}^{t} d s \int\left(e^{-\left|e^{-s Q} x\right|}-1\right) \rho_{c}(d x)\right] \int_{|z|<1} \exp \left[H_{c}(t, z)+I_{c}(t, z)\right] d z,
\end{aligned}
$$

where $I_{c}(t, z)=\int_{0}^{t} d s \int\left(e^{-\left|\left\langle z, e^{-s Q} x\right\rangle\right|}-e^{-\left|e^{-s Q} x\right|}\right) \rho_{c}(d x)$. Denote by $\mathbf{S}^{d-1}$ the $d-1$ dimensional unit sphere. Define a set $S_{Q}$ as

$$
S_{Q}=\left\{\xi \in \mathbf{S}^{d-1}:\left|u^{Q} \xi\right|>1 \quad \text { for } u>1\right\}
$$


We can disintegrate $\rho_{c}$ as

$$
\rho_{c}(E)=\int_{S_{Q}} \sigma(d \xi) \int_{0}^{\infty} 1_{E}\left(r^{Q} \xi\right) \tau_{\xi}(d r)
$$

for each Borel set $E$ in $\mathbf{R}^{d}$, where $\sigma$ is a probability measure on $S_{Q}$ and $\tau_{\xi}$ is a measure on $(0, \infty)$ such that $\tau_{\xi}(B)$ is measurable in $\xi$ for any Borel set $B$ in $(0, \infty)$ and $\tau_{\xi}((0, \infty))=\rho_{c}\left(\mathbf{R}^{d}\right)$. Hence setting $I(x)=e^{-|x|}$ in (i) of Lemma 3.1 and letting $e^{-s} r=\omega$, we obtain, for $z \in \mathbf{R}^{d}$ with $|z|<1$,

$$
\begin{aligned}
\left|I_{c}(t, z)\right| & =\int_{S_{Q}} \sigma(d \xi) \int_{0}^{\infty} \tau_{\xi}(d r) \int_{0}^{t} d s\left(e^{-\left|\left\langle z, e^{-s Q_{r} Q} \xi\right\rangle\right|}-e^{-\left|e^{-s Q} Q^{Q} \xi\right|}\right) \\
& \leq \int_{S_{Q}} \sigma(d \xi) \int_{0}^{\infty} \tau_{\xi}(d r) \int_{0}^{\infty} \frac{d \omega}{\omega}\left(e^{-\left|\left\langle z, \omega^{Q} \xi\right\rangle\right|}-e^{-\left|\omega^{Q} \xi\right|}\right) \\
& \leq \rho_{c}\left(\mathbf{R}^{d}\right)\left(K_{1}+K_{2} \int_{S_{Q}} \log \frac{1}{|\langle z, \xi\rangle|} \sigma(d \xi)\right)
\end{aligned}
$$

where $K_{1}$ and $K_{2}$ depend only on $Q$. From Lemma 2.2 of [7], choosing $c$ large enough, we have

$$
\begin{aligned}
& \int_{|z|<1} \exp \left[H_{c}(t, z)+I_{c}(t, z)\right] d z \\
& \leq \text { const } \int_{|z|<1} \exp \left[K_{2} \rho_{c}\left(\mathbf{R}^{d}\right) \int_{S_{Q}} \log \frac{1}{|\langle z, \xi\rangle|} \sigma(d \xi)\right] d z<\infty .
\end{aligned}
$$

Here we used Lemma 3.1 (ii) for $I(x)=2^{-1}\left(\cos x-e^{-|x|}\right)$. By virtue of Theorem 1 this theorem has been proved.

\section{§4. Example}

Now we present an example of Theorem 2. This example has been already treated in [7]. Let $X$ be an OU type process with $Q=\alpha I, \alpha>0$, and a Lévy measure $\rho$ such that, for every Borel set $E$ in $[0, \infty)$,

$$
\int 1_{E}(|x|) \rho(d x)=a \int_{E} \frac{d r}{r(\log (r \vee b))^{\gamma+1}},
$$

where $\gamma>0, a>0, b>1$. Then we have the following.

(i) If $\gamma>1, X$ is recurrent. 
(ii) If $\gamma<1, X$ is strongly transient.

(iii) If $\gamma=1$ and $a \leq \alpha, X$ is recurrent.

(iv) If $\gamma=1$ and $1<a / \alpha \leq 2, X$ is weakly transient.

(v) If $\gamma=1$ and $2<a / \alpha, X$ is strongly transient.

The conditions do not depend on the dimension $d$. K. Sato [5] proved that $d$-dimensional strongly non-lattice Lévy processes are strongly transient if $d \geq 5$. Hence this example shows the difference between Lévy processes and OU type processes.

Proof. Here we shall prove (ii), (iv), and (v). All the rest has been already proved in [7]. From now on let $c=b$. By the change of variables (1.3) becomes

$$
\begin{aligned}
& \int_{0}^{1} \frac{d v}{v} \log \frac{1}{v} \exp \left[\int_{v}^{1} \frac{d u}{u} \int\left(e^{-u^{\alpha}|x|}-1\right) \rho_{b}(d x)\right] \\
= & \frac{1}{\alpha^{2}} \int_{0}^{1} \frac{d v}{v} \log \frac{1}{v} \exp \left[-\frac{a}{\alpha} \int_{v}^{1} \frac{d u}{u} \int_{b}^{\infty} \frac{1-e^{-u r}}{r(\log r)^{\gamma+1}} d r\right] .
\end{aligned}
$$

Here we obtain

$$
\begin{aligned}
\int_{v}^{1} \frac{d u}{u} \int_{b}^{\infty} \frac{1-e^{-u r}}{r(\log r)^{\gamma+1}} d r & =\int_{v}^{1} d u \int_{b}^{\infty} \frac{d r}{r(\log r)^{\gamma+1}} \int_{0}^{r} e^{-u s} d s \\
& =\frac{1}{\gamma} \int_{v}^{1} d u \int_{b}^{\infty} \frac{e^{-u s} d s}{(\log s)^{\gamma}}+O(1) \\
& =\frac{1}{\gamma} \int_{b}^{2 / v} \frac{e^{-v s}}{s(\log s)^{\gamma}} d s+O(1)
\end{aligned}
$$

as $v \downarrow 0$.

(ii) If $\gamma<1$, then we get

$$
\int_{b}^{2 / v} \frac{e^{-v s}}{s(\log s)^{\gamma}} d s \geq \frac{e^{-2}}{1-\gamma}\left(\log \frac{2}{v}\right)^{1-\gamma}+O(1)
$$

as $v \downarrow 0$. Hence we see the right-hand side of (4.1) is finite.

(iv) If $\gamma=1$ and $1<a / \alpha \leq 2$, then we get

$$
\int_{b}^{2 / v} \frac{e^{-v s}}{s \log s} d s \leq \log \log \frac{2}{v}+O(1)
$$


as $v \downarrow 0$. Hence we see the right-hand side of (4.1) is infinite.

(v) If $\gamma=1$ and $2<a / \alpha$, then we get

$$
\int_{b}^{2 / v} \frac{e^{-v s}}{s \log s} d s \geq \int_{b}^{1 / v^{\beta}} \frac{e^{-v s}}{s \log s} d s
$$

for $v<1$, where $\beta=\beta(v)$ is a function of $v$ such that $0<\beta<1$. Then we have

$$
\begin{aligned}
\int_{b}^{1 / v^{\beta}} \frac{e^{-v s}}{s \log s} d s & \geq e^{-v^{1-\beta}} \int_{b}^{1 / v^{\beta}} \frac{d s}{s \log s} \\
& =e^{-v^{1-\beta}} \log \log \frac{1}{v^{\beta}}+O(1)
\end{aligned}
$$

as $v \downarrow 0$. We will calculate (4.1). Now choose $\delta$ small enough. Then

$$
\begin{aligned}
& \int_{0}^{\delta} \frac{d v}{v} \log \frac{1}{v} \exp \left[-\frac{a}{\alpha} \int_{v}^{1} \frac{d u}{u} \int_{b}^{\infty} \frac{1-e^{-u r}}{r(\log r)^{\gamma+1}} d r\right] \\
& \leq \int_{0}^{\delta} \frac{d v}{v} \log \frac{1}{v} \exp \left[-\frac{a}{\alpha} e^{-v^{1-\beta}} \log \log \frac{1}{v^{\beta}}+O(1)\right] \\
& \leq \operatorname{const} \int_{0}^{\delta} \frac{d v}{v} \exp \left[-\frac{a}{\alpha} e^{-v^{1-\beta}} \log \beta\right]\left(\log \frac{1}{v}\right)^{1-\frac{a}{\alpha} e^{-v^{1-\beta}}} \\
& \leq \text { const } \int_{0}^{\delta} \frac{d v}{v}\left(\log \frac{1}{v}\right)^{1-\frac{a}{\alpha} e^{-v^{1-\beta}}}
\end{aligned}
$$

Here we choose $\beta=\beta(v)$ such that $\beta(v) \rightarrow 1$ and $(1-\beta(v)) \log \frac{1}{v} \rightarrow \infty$ as $v \downarrow 0$. As a result, it follows from $a / \alpha>2$ that the last expression is finite.

Acknowledgements. The author thanks Professor K. Sato for suggesting the study of transient OU type processes. He would like to express his gratitude to Professor T. Shiga of Tokyo Institute of Technology for his valuable advice concerning Theorem 1 . The inequality (2.2.1) was suggested by him.

\section{REFERENCES}

[1] R. M. Blumenthal and R. K. Getoor, Markov Processes and Potential Theory, Academic Press, New York, 1968. 
[2] R. K. Getoor, Transience and recurrence of Markov processes, Lecture Notes in Math., Springer, 784 (1980), 397-409.

[3] S. C. Port, Limit theorems for transient Markov chains, J. Combinatorial Theory, 2 (1967), 107-128.

[4] S. C. Port and C. J. Stone, Infinitely divisible processes and their potential theory, I, II, Ann. Inst. Fourier, 21 (1971).

[5] K. Sato, Criteria of weak and strong transience for Lévy processes, Proceedings of Japan-Russia Symposium on Probability Theory and Mathematical Statistics (1995), World Scientific, 438-449.

[6] K. Sato and M. Yamazato, Operator-selfdecomposable distributions as limit distributions of processes of Ornstein-Uhlenbeck type, Stoch. Proc. Appl., 17 (1984), 73-100.

[7] K. Sato, T. Watanabe, and M. Yamazato, Recurrence conditions for multidimensional processes of Ornstein-Uhlenbeck type, J. Math. Soc. Japan, 46 (1994), 245-265.

[8] K. Sato, T. Watanabe, K. Yamamuro, and M. Yamazato, Multidimensional process of Ornstein-Uhlenbeck type with nondiagonalizable matrix in linear drift terms, Nagoya Math. J., 141 (1996), 45-78.

[9] T. Shiga, A recurrence criterion for Markov processes of Ornstein-Uhlenbeck type, Prob. Th. Rel. Fields, 85 (1990), 425-447.

[10] T. Watanabe, Sato's conjecture on recurrence conditions for multidimensional processes of Ornstein-Uhlenbeck type, J. Math. Soc. Japan, 50 (1998), 155-168.

Konan Women's Junior College

Takaya-cho, Konan 483

Japan 